\title{
Corrigendum: Emerging roles of ARHGAP33 in intracellular trafficking of TrkB and pathophysiology of neuropsychiatric disorders
}

Takanobu Nakazawa, Ryota Hashimoto, Kazuto Sakoori, Yuki Sugaya, Asami Tanimura, Yuki Hashimotodani, Kazutaka Ohi, Hidenaga Yamamori, Yuka Yasuda, Satomi Umeda-Yano, Yuji Kiyama, Kohtarou Konno, Takeshi Inoue, Kazumasa Yokoyama, Takafumi Inoue, Shusuke Numata, Tohru Ohnuma, Nakao Iwata, Norio Ozaki, Hitoshi Hashimoto, Masahiko Watanabe, Toshiya Manabe, Tadashi Yamamoto, Masatoshi Takeda \& Masanobu Kano

Nature Communications 7:10594 doi: 10.1038/ncomms10594 (2016); Published 03 Feb 2016; Updated 20 Apr 2016

Previous work by Rosário et al. 2007 and Schuster et al. 2015 describing the role of ARHGAP33 in regulating dendrite and dendritic spine development, as well as neuropsychiatric disorder-related behaviours in developing and adult mice, was inadvertently omitted from the reference list of this Article and should have been cited as follows. The statement in the Results section 'The roles of ARHGAP33 in the adult brain have not been investigated' should have read 'Previous work has investigated the role of ARHGAP33 in dendrite and dendritic spine development, as well as in autism-like behaviours (Rosário et al. 2007, Schuster et al. 2015, refs 6-9)'.

Rosário, M., Franke, R., Bednarski, C., \& Birchmeier, W. The neurite outgrowth multiadaptor RhoGAP, NOMA-GAP, regulates neurite extension through SHP2 and Cdc42. J. Cell. Biol. 178, 503-516 (2007).

Schuster, S., et al. NOMA-GAP/ARHGAP33 regulates synapse development and autistic-like behavior in the mouse. Mol. Psychiatry 20, 1120-1131 (2015).

(c) (i) This work is licensed under a Creative Commons Attribution 4.0 International License. The images or other third party material in this article are included in the article's Creative Commons license, unless indicated otherwise in the credit line; if the material is not included under the Creative Commons license, users will need to obtain permission from the license holder to reproduce the material. To view a copy of this license, visit http://creativecommons.org/licenses/by/4.0/ 